\title{
Votando al Partido Popular de Galicia: análisis de los componentes del voto a la formación en las elecciones autonómicas (1993-2016) ${ }^{1}$
}

\author{
Rubén Gómez Cebey \\ UNIVERSIDAD DE SANTIAGO DE COMPOSTELA, SANTIAGO DE COMPOSTELA, ESPAÑA \\ ruben.gomez.cebey@rai.usc.es
}

Resumen: Este trabajo analiza los componentes del voto al Partido Popular en las elecciones al Parlamento de Galicia celebradas entre 1993 y 2016. Profundiza en las principales tendencias que ha dibujado el comportamiento del electorado popular a lo largo del período autonómico atendiendo a los factores clásicos que han podido estar en su base, como la identificación partidista, la valoración del liderazgo, el contexto económico y político o la ideología. Este artículo presta, también, especial atención a la influencia del galleguismo, un factor que ha sido puesto en valor desde diferentes ámbitos, tanto académicos como políticos.

Palabras clave: Partido Popular, comportamiento electoral, componentes de voto, galleguismo, elecciones autonómicas, regresión logística.

Abstract: In this paper we analyze the components of the vote for the Partido Popular in the elections to the Galician Parliament held from 1993 to 2016. We delve into the main trends that the behavior of the popular electorate has drawn throughout the autonomous period, taking into account the different classics components of voting that may have been at its base, such as party identification, economic and political context or ideology. Finally, we will pay special attention to the influence that galleguismo has been able to exert on the behavior.

Key words: Partido Popular, electoral behavior, components of the vote, Galician nationalism, autonomic elections, logistic regression.

\section{Introducción}

as elecciones al Parlamento de Galicia, celebradas el 12 de julio de 2020, otorga-
ron al Partido Popular (PP) una nueva victoria y su octava mayoría absoluta en
la Cámara. En un contexto político complejo, marcado por la aparición de nue-
vos actores en la competición y después de una suspensión electoral —-derivada de la
crisis sanitaria de la covid-19 que marcó la agenda de campaña-, los populares fueron

1. Este artículo es el resultado de la adaptación del Trabajo Fin de Grado del autor, El comportamiento electoral de los votantes del Partido Popular de Galicia: de Fraga a Feijóo (1993-2016), dirigido por la Prof. María Pereira López, en el marco del Grado en Ciencias Políticas y de la Administración de la Universidad de Santiago de Compostela. 
capaces de obtener 42 de los 75 asientos en juego. Un resultado insólito para el partido en el conjunto de España, que reflejó la singularidad del caso gallego una vez más.

Sin embargo, para entender la hegemonía de la formación popular en Galicia no basta con atender a estas últimas elecciones, es preciso remontarse a los inicios del proceso de construcción de la autonomía. Fue en ese contexto cuando nacieron algunas de las singularidades que podrían haber acompañado a esta organización política hasta la actualidad, determinando su innegable éxito. Alianza Popular (AP) supo adaptarse entonces al nuevo marco de significados que emergía con el nacimiento de las instituciones autonómicas, fijando el futuro de la organización. Así lo demuestran los resultados de las elecciones inaugurales celebradas en 1981, que situaron al partido como la fuerza política más votada. Una victoria inesperada en un territorio en el que había ganado, hasta ese momento, la Unión de Centro Democrático (UCD) y que convirtió al aspirante de AP, Fernández Albor, en el primer presidente de la Xunta de Galicia.

La moción de censura liderada por el Partido Socialista Obrero Español (PSOE), que prosperó años más tarde, en 1987, contra el Gobierno de AP marcará el comienzo del final de una etapa. Se cerrará, definitivamente, con la victoria del PP en las elecciones de 1989. En esta ocasión, bajo el liderazgo de Manuel Fraga, la formación logrará su primera mayoría absoluta de escaños.

Estos resultados llevan consigo un cambio fundamental del sistema de partidos de Galicia. A partir de entonces, los populares comienzan a asentar su hegemonía en el ámbito del centro derecha. La consolidan junto a una de sus características fundamentales, el galleguismo. El también conocido como nacionalismo sui generis, nacionalismo blando, galleguismo templado o, simplemente, galleguismo, será uno de los factores diferenciales del PP en Galicia. No se puede entender el caso gallego — no sólo en lo que afecta a esta organización sino al conjunto del sistema de partidos políticos - , sin tener en cuenta esta dimensión.

Desde 1989, año en el que el Partido Popular de Galicia (PPdeG) consigue su primera mayoría absoluta, se sucederán otras tres más, en las elecciones de 1993, 1997 y 2001. Sólo en el año 2005 la formación popular no conseguirá sumar más escaños que el resto de los partidos con representación parlamentaria, permitiendo al PSOE y al Bloque Nacionalista Gallego (BNG) formar gobierno. Por segunda vez en la historia autonómica, Galicia tendrá un presidente de la Xunta de un partido distinto al PPdeG, si bien, al contrario de lo que había sucedido en 1987, en esta ocasión no será bajo el marco de una moción de censura.

Este breve impasse se resolverá satisfactoriamente para el PP en el año 2009. Bajo el nuevo liderazgo de Alberto Núñez Feijóo, los populares regresarán al Gobierno autonómico y volverán a sumar más asientos que el resto de los partidos políticos de la oposición en el hemiciclo, también en los comicios de 2012, 2016 y 2020.

Bajo este contexto y atendiendo a los resultados de las últimas elecciones autonómicas, surge una cuestión clave que vehiculará nuestro análisis ¿Cuáles han sido los factores que han resultado determinantes en la explicación del voto a esta formación en Galicia? 


\section{Objetivos y metodología}

Detrás de la pregunta que nos hemos realizado en el apartado anterior se encuentra el objetivo principal de nuestro trabajo, analizar los componentes del voto al PPdeG en el período 1993-2016. En definitiva, explicar qué factores se encuentran detrás de la decisión de votar a esta formación política y su peso diferencial, atendiendo a si éste ha variado en los diferentes procesos electorales o si, por el contrario, se dibuja algún patrón común.

Para nuestro propósito, hemos recurrido a los datos de siete estudios demoscópicos poselectorales elaborados por el Centro de Investigaciones Sociológicas (CIS) desde el año 1993 al 2016². Aunque nuestra estrategia de investigación atenderá a aspectos de carácter descriptivo, el grueso de nuestro trabajo recaerá en la elaboración de un análisis multivariante. Mediante el uso de modelos de regresión logística binaria podremos dar cuenta de los componentes del voto al partido. Para ello diseñaremos, previamente, un esquema propio, al que incorporaremos distintos factores explicativos que ha contemplado la literatura clásica sobre comportamiento electoral en este tipo de modelizaciones (Lagares, Jaráiz y Pereira, 2019; Rivera y Jaráiz, 2017; Lagares, Pereira y Rivera, 2018; Lagares, 2015)3. Las variables independientes empleadas se pueden ver en detalle en la tabla 1 . La variable dependiente será, en todos los casos, el hecho de haber votado al Partido Popular (votar PP), un factor que recodificaremos, previamente, a partir de la variable original de recuerdo de voto correspondiente.

Para cada elección realizaremos un planteamiento basado en modelos aditivos, de tal forma que procederemos agregando paulatinamente variables en un total de dos modelos de regresión a los que denominaremos modelo A y modelo B. A través del modelo A aislaremos el efecto de los cleavages políticos-escala ideológica e identitaria- sobre el voto, utilizando las variables sociodemográficas como variables de control. Mediante el modelo B trataremos de averiguar cuáles han sido los factores significativos para la explicación global del voto al $\mathrm{PP}^{4}$.

2. Los estudios consultados han sido: $3.155,2.963,2.796,2.611,2.434,2.263$ y 2.070 . Los datos relativos a estos estudios pueden ser consultados en la página web del CIS, de donde fueron extraídos: http://www.cis. es (recuperado el 10 de agosto de 2020). El procedimiento de muestreo de estos estudios varía ligeramente de unos a otros, en gran medida debido a la evolución en la forma de proceder del CIS y a su propio desarrollo como organización. Esto no nos impedirá realizar comparaciones, siempre con el debido cuidado, entre las distintas elecciones. Estamos, pues, ante una investigación con un enfoque metodológico cuantitativo y con un carácter analítico-longitudinal.

3. Por falta de homogeneidad entre los diferentes estudios postelectorales, en algunas de las regresiones no podremos introducir todas las variables. En algunos casos buscaremos alternativas, en otros, simplemente, será imposible sustituir las variables independientes por otras, por lo que procederemos a omitirlas.

4. En el modelo A se incluirá: escala de autoubicación ideológica, escala de autoubicación nacionalista, escala de ubicación ideológica del PP, escala de ubicación nacionalista del PP, pregunta Linz-Moreno, nivel de estudios, tamaño del municipio, sexo, edad y determinadas variables contextuales que aparecerán en algunos estudios. En el modelo B se introducirán todas las demás variables, incluyendo las del modelo A. 
El período temporal de nuestra investigación abarca las elecciones al Parlamento de Galicia celebradas entre 1993 y 2016, ambas incluidas. A priori, puede llamar la atención nuestra elección, puesto que no parece responder a un criterio concreto. La explicación se encuentra en la falta de datos disponibles para los comicios celebrados en 1981, 1985 y 1989. El CIS no ofrece las matrices correspondientes a los estudios de estas tres elecciones, lo que nos obliga a reducir el análisis.

Tabla 1

Variables independientes introducidas en los modelos de regresión

\begin{tabular}{|c|c|c|}
\hline & Variables & Operacionalización \\
\hline \multirow[t]{8}{*}{$\begin{array}{l}\text { Variables } \\
\text { contextuales }\end{array}$} & $\begin{array}{l}\text { Valoración de la situación } \\
\text { política actual de España }\end{array}$ & $\begin{array}{l}\text { Variable ordinal del tipo Likert } \\
\text { (muy buena, buena, regular, mala o muy } \\
\text { mala) }\end{array}$ \\
\hline & $\begin{array}{l}\text { Valoración de la situación } \\
\text { política actual de Galicia }\end{array}$ & $\begin{array}{l}\text { Variable ordinal del tipo Likert } \\
\text { (muy buena, buena, regular, mala o muy } \\
\text { mala) }\end{array}$ \\
\hline & $\begin{array}{l}\text { Valoración de la situación } \\
\text { económica actual de España }\end{array}$ & $\begin{array}{l}\text { Variable ordinal del tipo Likert } \\
\text { (muy buena, buena, regular, mala o muy } \\
\text { mala) }\end{array}$ \\
\hline & $\begin{array}{l}\text { Valoración de la situación } \\
\text { económica actual de Galicia }\end{array}$ & $\begin{array}{l}\text { Variable ordinal del tipo Likert } \\
\text { (muy buena, buena, regular, mala o muy } \\
\text { mala) }\end{array}$ \\
\hline & Valoración de la Xunta de Galicia & $\begin{array}{l}\text { Variable ordinal del tipo Likert } \\
\text { (muy buena, buena, regular, mala o muy } \\
\text { mala) }\end{array}$ \\
\hline & $\begin{array}{l}\text { Valoración de la labor llevada a cabo } \\
\text { por la Xunta en la financiación autonómica }\end{array}$ & $\begin{array}{l}\text { Variable ordinal del tipo Likert } \\
\text { (muy buena, buena, regular, mala o muy } \\
\text { mala) }\end{array}$ \\
\hline & $\begin{array}{l}\text { Valoración de la labor llevada a cabo } \\
\text { por la Xunta en la lengua gallega }\end{array}$ & $\begin{array}{l}\text { Variable ordinal del tipo Likert } \\
\text { (muy buena, buena, regular, mala o muy } \\
\text { mala) }\end{array}$ \\
\hline & $\begin{array}{l}\text { Valoración de la labor llevada a cabo } \\
\text { por la Xunta en el autogobierno de Galicia }\end{array}$ & $\begin{array}{l}\text { Variable ordinal del tipo Likert } \\
\text { (muy buena, buena, regular, mala o muy } \\
\text { mala) }\end{array}$ \\
\hline \multirow[t]{2}{*}{ Liderazgo } & Valoración de Fraga y Feijóo & $\begin{array}{l}\text { Variable de escala } 1-10 \\
\text { (1 mínima valoración y } 10 \text { máxima } \\
\text { valoración) }\end{array}$ \\
\hline & $\begin{array}{l}\text { Valoración de Beiras, Jorquera, Nogueira, } \\
\text { Leiceaga, Llamazares, Losada, Pontón, } \\
\text { Presedo, Quintana, Rajoy, Villares y Zapatero }\end{array}$ & $\begin{array}{l}\text { Variable de escala } 1-10 \\
\text { (1 mínima valoración y } 10 \text { máxima valoración) }\end{array}$ \\
\hline
\end{tabular}




\begin{tabular}{|c|c|c|}
\hline & Variables & Operacionalización \\
\hline $\begin{array}{l}\text { Identificación } \\
\text { partidista }\end{array}$ & Simpatía por el PP & $\begin{array}{l}\text { Variable ordinal del tipo Likert } \\
\text { (muy cercano, cercano, ni cercano ni } \\
\text { distante, distante o muy distante) }\end{array}$ \\
\hline \multirow[t]{6}{*}{$\begin{array}{l}\text { Ideología } \\
\text { política }\end{array}$} & $\begin{array}{l}\text { Autoubicación en la escala } \\
\text { ideológica (izq.-dcha.) }\end{array}$ & $\begin{array}{l}\text { Variable de escala } 1-10 \\
\text { (1 extrema izquierda y } 10 \text { extrema derecha) }\end{array}$ \\
\hline & $\begin{array}{l}\text { Autoubicación en la escala de } \\
\text { nacionalismo de Galicia }\end{array}$ & $\begin{array}{l}\text { Variable de escala } 1-10 \\
\text { ( } 1 \text { mínimo nacionalismo y } 10 \text { máximo } \\
\text { nacionalismo) }\end{array}$ \\
\hline & $\begin{array}{l}\text { Ubicación del PP en la escala } \\
\text { ideológica (izq.-dcha.) }\end{array}$ & $\begin{array}{l}\text { Variable de escala } 1-10 \\
\text { ( } 1 \text { extrema izquierda y } 10 \text { extrema derecha) }\end{array}$ \\
\hline & $\begin{array}{l}\text { Ubicación del PP en la escala de } \\
\text { nacionalismo de Galicia }\end{array}$ & $\begin{array}{l}\text { Variable de escala } 1-10 \\
\text { ( } 1 \text { mínimo nacionalismo y } 10 \text { máximo } \\
\text { nacionalismo) }\end{array}$ \\
\hline & $\begin{array}{l}\text { Sentimiento nacionalista de Galicia (se } \\
\text { siente únicamente español, se siente más } \\
\text { español que gallego, se siente tan español } \\
\text { como gallego, se siente más gallego que } \\
\text { español, se siente únicamente gallego) }\end{array}$ & $\begin{array}{l}\text { Variables dummy recodificadas a partir de la } \\
\text { variable original }\end{array}$ \\
\hline & Autodefinición como nacionalista gallego & $\begin{array}{l}\text { Variable dummy ( } 1 \text { definirse como } \\
\text { nacionalista gallego y } 0 \text { definirse como no } \\
\text { nacionalista gallego) a partir de la variable } \\
\text { nominal original }\end{array}$ \\
\hline \multirow[t]{2}{*}{$\begin{array}{l}\text { Campaña } \\
\text { electoral }\end{array}$} & Valoración de la campaña hecha por Fraga & $\begin{array}{l}\text { Variable ordinal del tipo Likert } \\
\text { (muy buena, buena, regular, mala o muy } \\
\text { mala) }\end{array}$ \\
\hline & Valoración de la imagen en campaña del PP & $\begin{array}{l}\text { Variable ordinal del tipo Likert } \\
\text { (muy buena, buena, regular, mala o muy } \\
\text { mala) }\end{array}$ \\
\hline \multirow{5}{*}{$\begin{array}{l}\text { Variables } \\
\text { socio- } \\
\text { demográficas }\end{array}$} & Sexo & $\begin{array}{l}\text { Variable dummy ( } 0 \text { ser hombre y } 1 \text { ser mujer) } \\
\text { recodificada a partir de la variable original }\end{array}$ \\
\hline & Edad & Escala de razón \\
\hline & Nivel de estudios & Variable ordinal \\
\hline & Nivel de ingresos & Variable ordinal \\
\hline & Tamaño del municipio & Variable ordinal \\
\hline
\end{tabular}




\section{Contexto: el Partido Popular de Galicia}

\subsection{El origen del Partido Popular de Galicia}

Las circunstancias que rodean el nacimiento del Partido Popular en Galicia marcan profundamente la estrategia de la formación, al menos, durante sus primeros años de vida. Los factores que resultan decisivos en la trayectoria del partido, de acuerdo con Lagares (1999: 89), pueden sintetizarse en cuatro: a) la existencia de una dimensión originaria centro-periferia; b) la imposibilidad de ocupar un espacio y un discurso político netamente centristas; c) el origen de cuadros o notables y d) la multiplicidad de asociaciones que conforman la primera coalición.

Atendiendo a sus argumentos, la creación del partido en Madrid como un centro político, permitirá que en Galicia el origen de AP se plantee, con matices, desde parámetros próximos a los de la fundación externa. En las tierras gallegas existía un clima favorable a la creación de una fuerza política continuista. Las tres organizaciones de mayor importancia en este proceso, por su implantación en el territorio, eran Acción Democrática Española (ADE), Unión Nacional Española (UNE) y Reforma Democrática (RD). Sin embargo, la presencia de estas organizaciones era desigual en las provincias gallegas y su vinculación a AP tenía más que ver con afinidades personales que con cuestiones estrictamente políticas.

Las debilidades de este modelo originario comenzaron a hacerse evidentes durante la redacción de la Constitución Española (CE). Bajo ese marco afloraron las primeras contradicciones internas entre los distintos equilibrios del partido. La posición de Manuel Fraga con respecto al debate de las nacionalidades y al Título VIII provocó una fractura en la organización, que se resolvió con la salida de algunos de los máximos dirigentes del partido, todavía reacios a la descentralización del Estado. Sin embargo, serán los pésimos resultados de las elecciones generales de 1979 -que llevan al partido al borde de la desaparición - los que realmente condicionen el cambio de rumbo de los populares. El fracaso electoral fue determinante para que los sectores más reaccionarios de AP abandonaran el partido propiciando, sin saberlo, el relevo generacional de los cuadros directivos y, con ello, un cambio en la estrategia.

\subsection{La primera victoria del Partido Popular}

Galicia no tenía tradición electoral cuando, al amparo de la Constitución Española de 1975, accedió al autogobierno. El comportamiento del electorado gallego en los comicios legislativos de 1977 y 1979, se había caracterizado por una baja participación y por un gran apoyo a las formaciones de derecha, encabezadas por la UCD. En las elecciones inaugurales de la etapa autonómica, celebradas el 20 de octubre de 1981, los partidos de la derecha se llevaron, como era previsible, la mayor parte de los 
votos. Sin embargo, lo que sobresalió de este primer encuentro con las urnas fue la inesperada victoria de AP, que superó en escaños a la UCD, el partido que hasta ese momento había dominado el panorama político gallego ${ }^{5}$. La autonomía gallega iba a ser construida y administrada por la fuerza política más reticente, hasta entonces, a la descentralización del Estado (Beramendi, 2016: 220). El partido que se había fracturado poco antes por las discrepancias respecto al Título VIII de la CE estaba llamado a gobernar Galicia.

La clave de este éxito se puede explicar por el desgaste sufrido por la UCD durante el proceso de aprobación del Estatuto de Autonomía, pero también tuvieron que ser determinantes otros factores, como los cambios estratégicos que se produjeron en el seno del partido después del fracaso de Coalición Democrática (CD) en las elecciones de 1979, tal y como observa Barreiro (2016: 13). Desde ese momento y hasta las elecciones de 1981, al mismo tiempo que los populares comenzaron a defender el hecho autonómico y el Estatuto de Autonomía, se inició el proceso de "galleguización" del partido.

Después de las primeras elecciones, el sistema político gallego ya había quedado definido por la singular presencia "una extraña formación de derecha que, para conservar su electorado en las grandes ciudades y en los núcleos rurales, tuvo que inventarse un nacionalismo sui generis y crear una estructura organizativa intensamente territorializada"6 (Barreiro, 2016: 11-13). De este modo, con un perfil marcado por el galleguismo, Gerardo Fernández Albor se convirtió en el primer presidente de la Xunta de Galicia. Sin embargo, debido a la inexperiencia política de la cabeza del ejecutivo, el peso de la acción política del Gobierno recaerá sobre el vicepresidente, Xosé Luís Barreiro ${ }^{7}$.

\subsection{La moción de censura, pasou o que pasou}

Después de la victoria de AP en Galicia, se inició un cambio de ciclo en toda España que tuvo como resultado la desaparición de la UCD. Un proceso de descomposición que AP aprovechó para acoger en su seno a gran parte de los antiguos miembros de la

5. En estas dos elecciones, el electorado gallego había seguido las mismas pautas de comportamiento que los votantes del resto de las Comunidades, algo que cambia con las autonómicas de 1981 (Rivera, Lagares, Castro y Diz, 1999: 300).

6. Con relación al nacionalismo sui generis, Rivera observa que el Partido Popular de Galicia manifiesta una tendencia nacional sensiblemente superior a la que el partido presenta en otras Comunidades Autónomas, lo que, sin duda, le ha permitido construir en torno al hecho diferencial, una marca de identidad propia (Rivera, 2003: 417).

7. El testimonio de Barreiro Rivas citado en varias ocasiones en este trabajo es especialmente valioso. Fue el mismo quien planificó y ejecutó algunos de los cambios en AP, para muchos es el auténtico constructor del partido en Galicia, por delegación de Manuel Fraga (Beramendi, 2016: 221). 
formación centrista y completar, así, una estructura basada en baronías provinciales que se reforzará a través del gobierno de las diputaciones" (Beramendi, 2016: 221) . $^{8}$

Es en este marco en el que surge Coalición Galega (CG), una organización política creada a partir de los restos de la UCD y que ocupó el campo del galleguismo de centroderecha. La presencia de este partido impedirá a los populares lograr la mayoría absoluta en el Parlamento en las elecciones de 1985. CG supuso un debilitamiento de AP debido a la atracción de personal político y votantes hacia el nacionalismo (Rivera, Lagares, Castro y Diz, 1999: 301). Nunca un partido ideológicamente situado en la derecha y con un discurso galleguista volvería a alcanzar un resultado similar.

Las elecciones de 1985 dejaron a la formación popular con 34 escaños, una mayoría suficiente para formar gobierno pero que, dada la heterogeneidad de la oposición, dificultó la investidura de Fernández Albor. Pese a todo, los verdaderos problemas para el partido aún estaban por llegar. El modelo consolidado desde la primera legislatura, en el que el hombre fuerte de la acción política era el vicepresidente, saltó por los aires. Las tensiones entre Fernández Albor y Barreiro Rivas desembocaron en la salida del ejecutivo del vicepresidente y de otros cinco diputados, que más tarde ingresaron en Coalición Galega. Todos los movimientos dieron lugar al Pacto de los Tilos y acabaron en la moción de censura que, el 23 de septiembre de 1987, hizo presidente de la Xunta de Galicia al socialista Fernando González Laxe. Sobre este episodio Manuel Fraga (1987: 452) llegó a declarar: "aquel día (...) tomé (...) una decisión (...): podré dejarlo todo, pero no mi obligación con mi Galicia, a la que tanto debo".

La llegada de Manuel Fraga trajo la primera mayoría absoluta de la historia autonómica gallega y del PP, marcando profundamente el sistema político de Galicia. El nuevo líder fue investido presidente gracias a una coalición preelectoral con Centristas de Galicia (CdeG), sólo en la circunscripción de Ourense. A pesar de la aparente estabilidad, sustentada en la mayoría absoluta, en 1991 se concretará la integración de CdeG en el PP. A través del Pacto de Lalín se alejarán así, los viejos fantasmas del pasado?.

Hay quien asume que Fraga siempre había estado presente en la política autonómica gallega, aludiendo a la famosa campaña "Galego como ti". Lo cierto es que su llegada a la Xunta de Galicia supuso un cambio sustancial, tanto en el sistema político como en su propio partido, desplazando la formación hacia posiciones aún más properiféricas (Lagares, 1999, Lagares, 2003 y Lugilde, 2013).

8. Este modelo, que mantendrá su vigencia, al menos, hasta la llegada de Núñez Feijóo a la presidencia de la Xunta de Galicia, mostrará sus debilidades con claridad en la última legislatura de Manuel Fraga.

9. Este no es un asunto menor, con la entrada de la formación Centristas de Galicia en el seno del Partido Popular, se culmina un proceso de cambio organizativo que había comenzado a partir de la descomposición de la UCD que supone 1) la culminación del proceso de asimilación de fuerzas políticas próximas y 2) el desplazamiento de la organización popular hacia el centro del espectro político, lo que posibilita la representación de un galleguismo moderado (Lagares, 1999). 


\section{Análisis de resultados}

\subsection{Dimensiones espaciales en la política gallega}

Atendiendo al marco previo, parece evidente que para la articulación de las preferencias de los ciudadanos, en general, y de los votantes populares, en particular, en Galicia no sólo es fundamental atender a la variable ideológica, sino también a la nacionalista (Rivera, 2003 y Ares y Rama, 2019). Del mismo modo, en el caso del sistema político gallego resulta imprescindible tener en consideración el componente galleguista del PP, un factor que refuerza todavía más la importancia de la dimensión identitaria.

Frente al relativo acuerdo sobre la existencia de esta particularidad en la organización popular, la multiplicidad de nombres con los que se ha abordado nos muestra la ausencia de una definición de consenso por parte de la literatura que ha tratado la cuestión. De acuerdo con Lagares (1999; 2003), son cuatro los principales argumentos que nos permiten hablar de galleguismo en el PP, al menos, en los primeros años de la autonomía: a) la independencia de la organización popular en Galicia con respecto al partido en Madrid; b) el liderazgo de Fraga; c) las dinámicas de integración de fuerzas nacionalistas; $\mathrm{y}$ d) el uso de símbolos que ha contribuido a reforzar la idea del hecho diferencial y el proceso de identificación del partido con los intereses de Galicia.

$\mathrm{Al}$ arranque de la autonomía, la AP gallega consiguió un alto nivel de independencia orgánica por su condición de partido de gobierno. Esto obligó a los populares a adecuar su discurso a la nueva realidad autonómica de una forma específica y a dar contenido político a la nueva instancia administrativa. La capacidad de tomar decisiones al margen de la ejecutiva estatal permitirá a la organización gallega funcionar de acuerdo a sus propios criterios, aunque en ocasiones éstos puedan chocar con los del aparato del partido en Madrid (Lagares, 1999). En este sentido, será el liderazgo de Manuel Fraga el que legitime la evolución hacia presupuestos autonomistas, permitiendo que este proceso se realice de una manera natural, evitando confrontaciones con la estrategia de AP en el ámbito estatal. Ya como presidente de la Xunta de Galicia, su postura llegará a estar próxima a agotar las posibilidades autonómicas de Galicia al proponer una Administración Única (Lagares, 1999 y 2003; Fraga, 1993). Durante sus gobiernos existirán dudas sobre si el PPdeG se podría considerar, incluso, como una formación de ámbito no estatal (Rivera, Lagares, Castro y Diz, 1999).

Otro de los factores que determina el cambio organizativo del PP hacia la "galleguización" es la integración de fuerzas nacionalistas. Como ya destacamos, este proceso culmina con la entrada de CdeG. Lagares (1999) destaca que esto se traducirá en un desplazamiento de la organización popular hacia posiciones del centro del espectro, ocupando el espacio dejado por los anteriores partidos de corte galleguista. Todo lo anterior se refuerza, finalmente, por el uso de símbolos tradicionales de Galicia, que habrían contribuido a reforzar la idea del hecho diferencial y al proceso de 
identificación del partido con los intereses de Galicia (Lagares, 1999 y 2003). En este ámbito destaca el papel de la Xunta de Galicia, especialmente a través de la Radio Televisión de Galicia, a la hora de difundir el idioma, las costumbres, los símbolos y las tradiciones particulares del pueblo gallego. No parece difícil imaginar que, después de años de Gobierno - sólo interrumpido en dos ocasiones- se produzca una identificación entre la estructura de la Xunta de Galicia y la del partido en la defensa de aquellos valores culturales propios, que aparecen vinculados a la tradición (Lagares, 2003: 72-73). De este modo, la valoración que los electores hagan del Gobierno gallego será fundamental a la hora de explicar el voto al PP en Galicia.

Aunque se alimenta y acomoda a las percepciones de los ciudadanos, el galleguismo del PP es endógeno a la organización y será una parte fundamental de la estrategia del partido, al menos, durante las primeras elecciones autonómicas y a la llegada de Manuel Fraga a la Comunidad.

\subsubsection{Autoubicación de los votantes en la escala ideológica y nacionalista}

Desde 1993 hasta la actualidad, los votantes del PPdeG se han ubicado, de media, en posiciones más a la derecha que el conjunto de los electores en las elecciones autonómicas, si bien, es posible observar cambios. Mientras que en los comicios de 1993 la autoubicación ideológica media de los votantes del partido era de 7,4, el máximo; en el año 2009 ésta había descendido hasta 6,53, el mínimo ${ }^{10}$. De todos modos, es entre las elecciones de 1993 y el resto de los comicios donde se observa el mayor contraste en las posiciones de los votantes del PPdeG.

Respecto a la autoubicación en la escala nacionalista, los votantes del PPdeG se han situado, de media, en posiciones más escoradas hacia la parte no nacionalista. A nivel descriptivo podemos atender a dos cambios bruscos en la ubicación dentro de esta escala: el primero, entre el año 1997 y el año 2001; y, el segundo y más llamativo, entre el año 2001 y el 2005, cuando las posiciones pasaron de situarse, de media, en el 4,75 a hacerlo en el 3,60. Dicho de otro modo, entre las elecciones de 1997 y las de 2005, los votantes del PP pasaron de ubicarse en el 5,67 a hacerlo en el 3,60, un cambio nada despreciable a nivel descriptivo ${ }^{11}$.

10. En todos los casos dentro de una escala 1-10, donde el 1 corresponde a extrema izquierda y el 10 a extrema derecha.

11. En todos los casos dentro de una escala 1-10, donde el 1 corresponde a máximo no nacionalismo y el 10 a máximo nacionalismo. 
Tabla 2

Autoubicación de los votantes gallegos y de los votantes del Partido Popular en la escala ideológica y nacionalista entre los años 1993 y 2016

\begin{tabular}{|l|l|c|c|c|c|c|c|c|}
\hline \multicolumn{2}{|c|}{} & $\mathbf{1 9 9 3}$ & $\mathbf{1 9 9 7}$ & $\mathbf{2 0 0 1}$ & $\mathbf{2 0 0 5}$ & $\mathbf{2 0 0 9}$ & $\mathbf{2 0 1 2}$ & $\mathbf{2 0 1 6}$ \\
\hline \multirow{2}{*}{$\begin{array}{l}\text { Escala de autoubica- } \\
\text { ción ideológica (1-10) }\end{array}$} & $\begin{array}{l}\text { Total } \\
\text { muestra }\end{array}$ & 5,29 & 5,29 & 5,43 & 4,95 & 4,92 & 4,98 & 4,88 \\
\cline { 2 - 10 } & Votante PP & 7,40 & 6,84 & 6,93 & 7,08 & 6,53 & 6,96 & 6,79 \\
\hline \multirow{2}{*}{$\begin{array}{l}\text { Escala de autoubi- } \\
\text { cación nacionalista } \\
(1-10)\end{array}$} & $\begin{array}{l}\text { Total } \\
\text { muestra }\end{array}$ & 5,81 & 5,88 & 5,09 & 4,45 & 4,46 & 4,22 & 4,28 \\
\cline { 2 - 10 } & Votante PP & 5,54 & 5,67 & 4,75 & 3,60 & 3,87 & 3,83 & 3,79 \\
\hline
\end{tabular}

Fuente: elaboración propia a partir de los datos del CIS.

A priori podemos extraer dos conclusiones respecto de estos datos: 1) a grandes rasgos, los votantes del PP se han desplazado a posiciones más centrales dentro de la escala de autoubicación ideológica, aunque sin descender nunca del 6,53; y, 2) la posición media en la escala identitaria, de los que han votado al PP entre 1993 y 2016, se ha situado en el lado no nacionalista, exceptuando 1993 y 1997, coincidiendo en este último caso, con el mayor nivel de competición política con su principal rival, nacionalista además, el BNG.

Lo expuesto anteriormente lo podemos observar, de una forma clara, en los siguientes gráficos de dispersión, los cuales representan la autoubicación de los votantes del PP en el espacio definido por la variable ideológica (izquierda/ derecha) y la variable nacionalista (no nacionalismo/ nacionalismo) en 1993 y $2016^{12}$.

En concreto, en el gráfico 1, vemos que la formación popular ocupa todo el espacio de centroderecha de forma hegemónica, una de las características del sistema político gallego desde las elecciones de 1989. Si comparamos los resultados que obtenemos para las elecciones de 1993 con los de 2016, encontramos un cambio sustancial que afecta a la escala identitaria. En contraste a lo que refleja el gráfico en 1993, en 2016 el cuadrante correspondiente a la dimensión derecha/ nacionalismo se vacía

12. Como la agrupación de los casos se ha realizado por tamaño, cuanto mayores son los puntos, mayor es el número de casos que se acumulan en ellos y viceversa. Por el enorme espacio que se necesita para poder apreciar en detalle toda la información descriptiva que nos aportan los gráficos de dispersión, sólo atenderemos a algunos por su especial interés. 
casi por completo. Junto a esto, de manera absolutamente gráfica, podemos ver concentraciones de votantes que se sitúan en el extremo no nacionalista, algo que a nivel descriptivo no sucedía en 1993.

\section{Gráfico 1}

Autoubicación en los ejes ideológico y nacionalista de los votantes del PP en 1993 y 2016

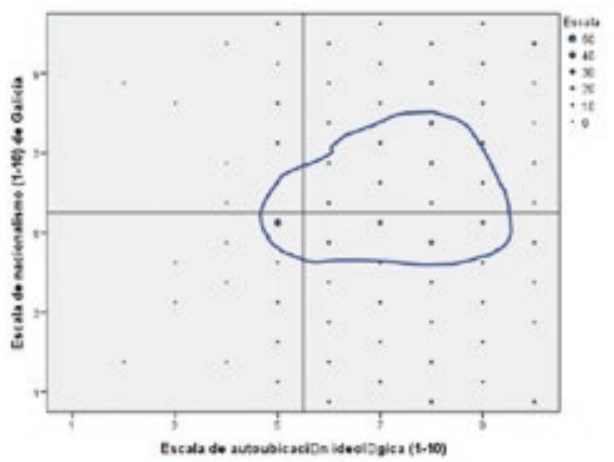

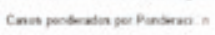

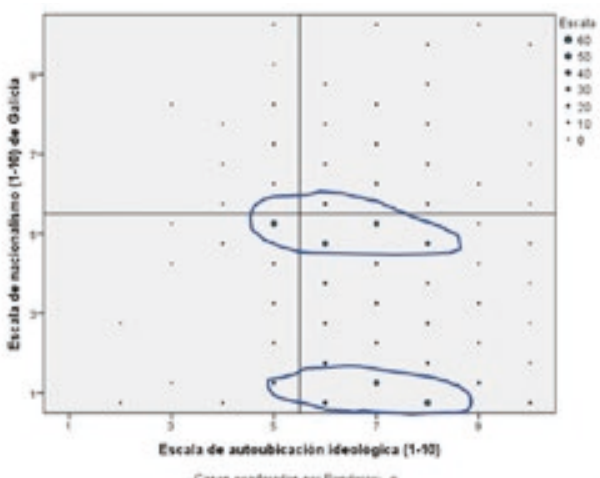

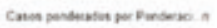

Fuente: elaboración propia a partir de datos del CIS.

\subsubsection{Ubicación subjetiva del Partido Popular en la escala ideológica y nacio-} nalista

Habiendo observado la autoubicación de los electores en la escala ideológica y nacionalista, nos centramos, a continuación, en cómo los votantes han ubicado y por tanto percibido, al PPdeG.

Comenzando por la escala ideológica, podemos poner de relieve tres aspectos: 1) el total de los electores gallegos ha situado, de media, al PP en posiciones superiores a 7,63, habiéndose producido el máximo en las elecciones de 2016 (8,3 de media);2) los votantes populares perciben al partido en posiciones más centrales que el conjunto de los votantes ${ }^{13}$; y, 3) los electores de la formación se ubican a sí mismos más a la izquierda de lo que ubican a su propio partido. 
Tabla 3

Ubicación subjetiva del Partido Popular en la escala ideológica y nacionalista entre los años 1993 y 2016

\begin{tabular}{|c|l|r|r|r|r|r|r|r|}
\hline \multicolumn{2}{|c|}{} & 1993 & 1997 & 2001 & 2005 & 2009 & 2012 & 2016 \\
\hline $\begin{array}{c}\text { ESCALA DÉ } \\
\text { UBICACIÓ } \\
\text { IDEOLÓGIC } \\
\text { A (1-10) PARA } \\
\text { EL PP }\end{array}$ & $\begin{array}{l}\text { Total } \\
\text { muestra }\end{array}$ & 8,10 & 7,63 & & 7,90 & 7,88 & 8,26 & 8,3 \\
\hline $\begin{array}{c}\text { ESCALA DE } \\
\text { NACIONAL } \\
\text { SMO (1-10) } \\
\text { PARA EL PP }\end{array}$ & $\begin{array}{l}\text { Total } \\
\text { muestra }\end{array}$ & 4,11 & 7,52 & & 7,77 & 7,5 & 7,9 & 7,93 \\
\hline
\end{tabular}

Fuente: elaboración propia a partir de los datos del CIS.

Por otro lado, con respecto a la escala de nacionalismo gallego, podemos extraer que: 1) los votantes del PP ubican al partido en posiciones más hacia el lado nacionalista de la escala que el total de los electores; y 2), que con el paso de las elecciones los electores del PP lo perciben como menos nacionalista, pasando de 5,97 en 1993 a 3,59 en 2016.

Del gráfico 2, que representa cómo los electores del PP ubican a la organización en los años 1993 y 2016, destacan dos cuestiones. Primero, en ambos casos y en concordancia con lo que venimos observando, los electores populares perciben a su partido como una organización que ocupa todo el espacio de la derecha. Y segundo, los cambios en la percepción del componente galleguista, tan importante para el partido por parte de su electorado. Los resultados de 1993 contrastan con los que obtenemos para el año 2016. En este último caso vemos una importante concentración de votantes que perciben y sitúan a la organización popular en el extremo no nacionalista, algo que no sucedía en 1993. Junto a esto, las partes superiores del cuadrante derecha/ nacionalista, se vacían.

La pregunta obvia que cabe hacerse es si se ha perdido el componente galleguista, que habría caracterizado al partido en sus primeros años. Responder a esta cuestión no es tarea fácil, pero a nivel descriptivo, todo parece indicar que ha habido una importante rebaja en la percepción del galleguismo en la imagen del PP por parte de sus electores. Aunque, a través del análisis del contenido de los programas electorales, no se observa una disminución objetiva en las posiciones territoriales desde 1993 hasta 2016 (Ares y Rama, 2019), cuando atendemos a las percepciones de los votantes observamos una clara tendencia descendente en la ubicación media en la escala nacionalista. Esto se hace especialmente notable entre las elecciones de 1997 
y 2001, cuando pasa de 5,41 a 4,91; y entre las de 2001 y 2005, cuando desciende de 4,91 a 3,98 .

\section{Gráfico 2}

Ubicación subjetiva del PP en los ejes ideológico y nacionalista en 1993-2016

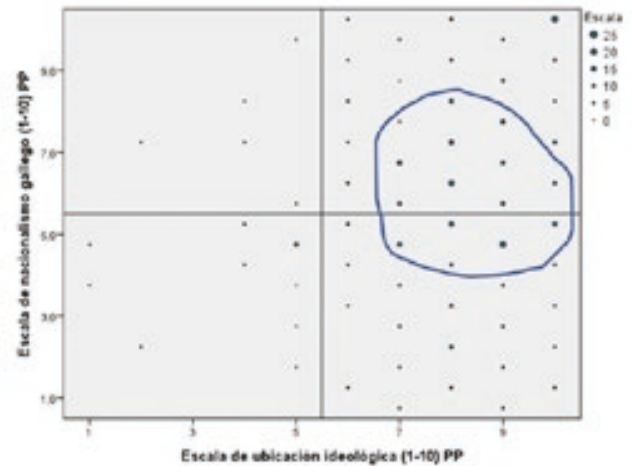

Cam punterstes pas hendersi.

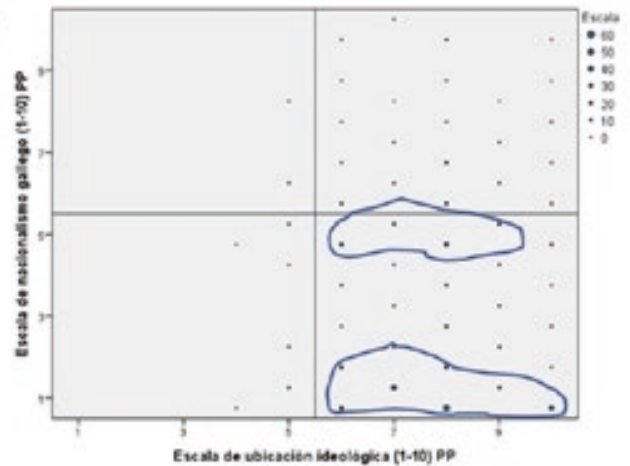

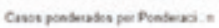

Fuente: elaboración propia a partir de datos del CIS.

\subsection{Componentes del voto del Partido Popular de Galicia}

Para cerrar nuestro análisis y centrándonos en el objetivo principal de este trabajo atendemos, ahora, a los factores que han resultado estadísticamente significativos en la explicación del voto al PP en las elecciones al Parlamento de Galicia celebradas entre 1993 y 2016. Lo hacemos través de nuestros modelos de regresión logística binaria, cuyos principales hallazgos se pueden observar, de manera sistemática y sintética, en las tablas 4 y $5^{14}$.

Comenzamos poniendo el foco sobre el eje ideológico - en su operacionalización como escala de autoubicación (1-10)-. Observamos que esta variable ha resultado significativa en todos nuestros modelos. Esto nos advierte de la importancia que este factor ha tenido históricamente en la explicación del voto al PP en nuestra comunidad. Como cabría esperar, la autoubicación de los votantes en posiciones de derecha ha aumentado las probabilidades de votar al PP. 
Frente a esto, la variable ubicación subjetiva del PPdeG en la escala ideológica sólo resulta significativa a partir de las elecciones de 2005 y en el modelo A, que recoge de entrada un menor número de variables. Además, y al contrario de lo que sucedía en el caso de la autoubicación ideológica, ubicar al PP en posiciones más a la derecha disminuye las probabilidades de votar a la formación. Como podemos observar, la relación que se produce entre cada una de estas variables - la autoubicación y ubicación del PPdeG en la escala ideológica - con respecto al voto resulta paradójica e incluso contradictoria y parece esconder algunas de las singularidades del partido a la hora de definir se estrategia en el espacio de competición.

Algo similar sucede en el caso de las variables ubicación y autoubicación del PPdeG en la escala nacionalista (1-10). En este caso, por un lado, según aumenta la autoubicación a posiciones de máximo nacionalismo se reducen las probabilidades de votar al PP y, por otro lado, según aumenta la ubicación de la formación a posiciones de máximo nacionalismo se incrementan las posibilidades de votar al partido. La presencia de estos factores no es constante en nuestros modelos. En el caso de la escala de autoubicación nacionalista, desaparece en las elecciones de 2005, 2012 y 2016 en el modelo A, mientras que en el B sólo resulta significativa en 1993, 1997 y 2001. La escala de ubicación nacionalista del PP aparece en todas las elecciones en el modelo A, a excepción de las del año 2005. Aunque este último factor no aparece en nuestro modelo B para los años 2005, 2012 y 2016, sin duda su presencia ha sido fundamental, al menos, hasta el año 2001. La relación que se produce entre estas dos escalas, con respecto al voto, puede entenderse mejor si ponemos el foco sobre el desprecio por el concepto nacionalista que el PPdeG ha desarrollado. Un rechazo que se combina, sin embargo, con la apuesta por el desarrollo de las competencias autonómicas y la defensa de los valores propios de Galicia, como su lengua y su cultura tradicional. En definitiva, estamos ante ese galleguismo que ha alejado al PPdeG en ocasiones de los postulados más centristas de la organización en Madrid.

En lo relativo a la identificación partidista, cabe destacar que este factor ejerce un impacto considerable en la explicación del voto al PP, incluso por encima de la valoración de los líderes. Como se observa en la tabla 5, según aumenta la simpatía por el partido mayor es la probabilidad de votarlo, lo que concuerda tanto con los estudios clásicos como con los más actuales sobre comportamiento electoral (Campbell, Converse, Miller y Stokes, Harrop y Miller, 1987 y Lewis-Beck, Norpoth y Jacoby, 2008). Llama la atención, eso sí, que la simpatía por el PP no resulte significativa en las elecciones de 1993, de todos modos, esto puede estar relacionado con el proceso de institucionalización en el que aún estaba inmersa la formación. 
En lo relativo a las variables contextuales, la valoración de la Xunta de Galicia ha tenido un peso especialmente relevante. Por su propia naturaleza, este factor ejerce efectos positivos o negativos sobre el voto al PP dependiendo de si éste se encontraba o no en el Gobierno. Así, en las elecciones del año 2009, una alta valoración de la Xunta disminuía las probabilidades de votar a los populares. Cabe subrayar que ni la situación económica ni política actual de Galicia resultan significativas en la explicación del voto en nuestro esquema.

La valoración de los líderes del PPdeG también juega un papel relevante ${ }^{15}$. La de Feijóo aparece como un factor estadísticamente significativo en las elecciones de 2009, 2012 y 2016, siempre ejerce un efecto positivo sobre el voto. También la de Manuel Fraga, aunque no existan datos para 1997 y 2001. Como se observa en la tabla 5, también han tenido relativa importancia la valoración de otros líderes como Mariano Rajoy y resulta subrayable que sea la valoración de los liderazgos del BNG la que realmente confronte con el PP.

Sin ejercer demasiado peso en la explicación del voto, las variables de orden sociodemográfico nos aportan información sobre distintas pautas. Por un lado, la edad ejerce un efecto positivo sobre el voto al PP - siempre que resulta significativa-. La explicación simplista y popularmente extendida, que relaciona las victorias del PPdeG con el voto de los ciudadanos de avanzada edad, no tiene demasiado sustento. Aunque el aumento de la edad explica el hecho de votar al partido en algunas ocasiones, como demuestran los resultados, en ningún caso resulta un factor clave. Por otro lado, el tamaño del municipio, medido en el número de habitantes, ha ejercido un efecto inverso en las probabilidades de votar a la formación popular en algunos modelos. Es verosímil que estos hallazgos, conocidos por el partido, hayan motivado estrategias como la de convertir en candidatos de las siete grandes ciudades a políticos que previamente habían ejercido su labor como conselleiros en el Gobierno autonómico. Finalmente, el nivel de ingresos y el nivel de estudios también aparece en alguno de los modelos, en todos los casos con signo negativo, es decir, según aumentan los niveles de ingresos y de estudios, en cada caso, disminuyen las probabilidades de votar al PP.

15. Hay que tener en cuenta que esta variable no estaba disponible en algunos modelos. 
Tabla 4

Variables significativas en el modelo $\mathrm{A}^{16}$

\begin{tabular}{|c|c|c|c|c|c|c|c|}
\hline Variables & 1993 & 1997 & 2001 & 2005 & 2009 & 2012 & 2016 \\
\hline $\begin{array}{l}\text { Escala de autoubi- } \\
\text { cación } \\
\text { ideológica }\end{array}$ & $\begin{array}{l}1,260 * * * \\
(0,104)\end{array}$ & $\begin{array}{l}1,352 * * * \\
(0,092)\end{array}$ & $\begin{array}{l}1,345 * * * \\
(0,090)\end{array}$ & $\begin{array}{l}1,729 * * * \\
(0,149)\end{array}$ & $\begin{array}{l}1,368 * * * \\
(0,092)\end{array}$ & $\begin{array}{l}1,494 * * * \\
(0,115)\end{array}$ & $\begin{array}{l}1,461 * * * \\
(0,142)\end{array}$ \\
\hline $\begin{array}{l}\text { Escala de nacionalis- } \\
\text { mo de Galicia }\end{array}$ & $\begin{array}{l}-0,371 * * * \\
(0,073)\end{array}$ & $\begin{array}{l}-0,214 * * * \\
(0,060)\end{array}$ & $\begin{array}{l}-0,442 * * * \\
(0,062)\end{array}$ & & $\begin{array}{l}-0,288 * * * \\
(0,054)\end{array}$ & & \\
\hline $\begin{array}{l}\text { Escala de ubicación } \\
\text { ideológica PP }\end{array}$ & & & $x$ & $\begin{array}{l}-0,306 * * * \\
(0,103)\end{array}$ & $\begin{array}{l}-0,181 * * * \\
(0,066)\end{array}$ & $\begin{array}{l}-0,226 * * \\
(0,091)\end{array}$ & $\begin{array}{l}-0,559 * * * \\
(0,117)\end{array}$ \\
\hline $\begin{array}{l}\text { Escala da nacionalis- } \\
\text { mo gallego PP }\end{array}$ & $\begin{array}{l}0,420 * * * \\
(0,066)\end{array}$ & $\begin{array}{l}0,386 * * * \\
(0,056)\end{array}$ & $\begin{array}{l}0,506 * * * \\
(0,054)\end{array}$ & & $\begin{array}{l}0,363 * * * \\
(0,057)\end{array}$ & $\begin{array}{l}0,296 * * * \\
(0,063)\end{array}$ & $\begin{array}{l}0,239 * * * \\
(0,078)\end{array}$ \\
\hline $\begin{array}{l}\text { Autodefinición como } \\
\text { nacionalista }\end{array}$ & $x$ & $x$ & $x$ & $\begin{array}{l}-0,810 * * \\
(0,353)\end{array}$ & & & \\
\hline $\begin{array}{l}\text { Más gallego que } \\
\text { español }\end{array}$ & & $\begin{array}{l}-0,776 * * * \\
(0,256)\end{array}$ & & & & & \\
\hline Únicamente español & $\begin{array}{l}-2,360 * * * \\
(0,747)\end{array}$ & & & $\begin{array}{l}1,961 * * \\
(0,908)\end{array}$ & & & \\
\hline $\begin{array}{l}\text { Valoración de la labor } \\
\text { de la Xunta de Galicia } \\
\text { en el autogobierno }\end{array}$ & $x$ & $x$ & $x$ & $x$ & $\begin{array}{l}-0,457 * * * \\
(0,153)\end{array}$ & $x$ & $\begin{array}{l}0,687 * * * \\
(0,233)\end{array}$ \\
\hline $\begin{array}{l}\text { Valoración de la Xunta } \\
\text { de Galicia }\end{array}$ & $\mathrm{x}$ & $x$ & $x$ & $\begin{array}{l}2,139 * * * \\
(0,239)\end{array}$ & $\begin{array}{l}-0,961 * * * \\
(0,167)\end{array}$ & $\begin{array}{l}1,475 * * * \\
(0,179)\end{array}$ & $\begin{array}{l}0,876 * * * \\
(0,252)\end{array}$ \\
\hline Edad & $\begin{array}{l}0,020 * \\
(0,008)\end{array}$ & $\begin{array}{l}0,017 * * * \\
(0,006)\end{array}$ & & $\begin{array}{l}0,019 * * \\
(0,009)\end{array}$ & & $\begin{array}{l}0,023 * * * \\
(0,008)\end{array}$ & \\
\hline Nivel de ingresos & $x$ & $x$ & $x$ & $x$ & $x$ & & $\begin{array}{l}-0,184 * * \\
(0,082)\end{array}$ \\
\hline Nivel de estudios & & & $\begin{array}{l}-0,199 * * * \\
(0,074)\end{array}$ & & & & \\
\hline Tamaño del municipio & & & $\begin{array}{l}-0,246 * * * \\
(0,084)\end{array}$ & & $\begin{array}{l}-0,188 * * \\
(0,089)\end{array}$ & & \\
\hline Constante & $-7,114$ & $-7,930$ & $-5,651$ & $-15,075$ & $-1,016$ & $-12,253$ & $-8,002$ \\
\hline $\begin{array}{l}\text { R cuadrado de Nage- } \\
\text { lkerke }\end{array}$ & 0,806 & 0,743 & 0,742 & 0,867 & 0,783 & 0,846 & 0,846 \\
\hline $\begin{array}{l}\text { Logaritmo de la vero- } \\
\text { similitud }\end{array}$ & 353,303 & 591,506 & 719,563 & 314,319 & 665,466 & 438,042 & 290,250 \\
\hline
\end{tabular}

Fuente: elaboración propia a partir de datos del CIS.

16. Para la interpretación de las tablas 18 y 19 es necesario tener en cuenta que las casillas marcadas con una " $x$ " $y$ de color gris indican que en ese año la variable en cuestión no estaba disponible; las casillas en blanco señalan que la variable fue introducida en el modelo, pero no resultó significativa. Así mismo, hay que tener en cuenta que el valor que aparece en cada casilla, con signo negativo o positivo, se corresponde con el coeficiente beta, mientras que el que aparece justo debajo, entre paréntesis, es el error estándar asociado al mismo. 
Tabla 5

Variables significativas en el modelo B

\begin{tabular}{|c|c|c|c|c|c|c|c|}
\hline Variables & 1993 & 1997 & 2001 & 2005 & 2009 & 2012 & 2016 \\
\hline $\begin{array}{l}\text { Escala de autoubica- } \\
\text { ción } \\
\text { ideológica }\end{array}$ & $\begin{array}{l}0,882 * * * \\
(0,122)\end{array}$ & $\begin{array}{l}1,336 * * * \\
(0,102)\end{array}$ & $\begin{array}{l}1,179 * * * \\
(0,094)\end{array}$ & $\begin{array}{l}0,769 * * * \\
(0,195)\end{array}$ & $\begin{array}{l}0,694 * * * \\
(0,127)\end{array}$ & $\begin{array}{l}0,820 * * * \\
(0,143)\end{array}$ & $\begin{array}{l}1,367 * * * \\
(0,249)\end{array}$ \\
\hline $\begin{array}{l}\text { Escala de nacionalismo } \\
\text { de Galicia }\end{array}$ & $\begin{array}{l}-0,296 * * * \\
(0,093)\end{array}$ & $\begin{array}{l}-0,163 * * \\
(0,065)\end{array}$ & $\begin{array}{l}-0,367 * * * \\
(0,068)\end{array}$ & & & & \\
\hline $\begin{array}{l}\text { Escala de ubicación } \\
\text { ideológica PP }\end{array}$ & & & $x$ & & & & \\
\hline $\begin{array}{l}\text { Escala da nacionalismo } \\
\text { gallego PP }\end{array}$ & $\begin{array}{l}0,321 * * * \\
(0,093)\end{array}$ & $\begin{array}{l}0,323 * * * \\
(0,063)\end{array}$ & $\begin{array}{l}0,381 * * * \\
(0,059)\end{array}$ & & $\begin{array}{l}0,178 * * \\
(0,088)\end{array}$ & & \\
\hline $\begin{array}{l}\text { Autodefinición como } \\
\text { nacionalista }\end{array}$ & $x$ & $x$ & $x$ & & $\begin{array}{l}-1,323 * * * \\
(0,424)\end{array}$ & & \\
\hline $\begin{array}{l}\text { Más gallego que } \\
\text { español }\end{array}$ & & $\begin{array}{l}-0,881 * * * \\
(0,277)\end{array}$ & & & & & \\
\hline $\begin{array}{l}\text { Valoración de la labor } \\
\text { de la Xunta en el } \\
\text { autogobierno }\end{array}$ & $x$ & $\mathrm{x}$ & $\mathrm{x}$ & $x$ & & $\mathrm{x}$ & \\
\hline $\begin{array}{l}\text { Valoración de la Xunta } \\
\text { de Galicia }\end{array}$ & $x$ & $\mathrm{x}$ & $\mathrm{x}$ & $\begin{array}{l}1,790 * * * \\
(0,411)\end{array}$ & $\begin{array}{l}-0,727 * * * \\
(0,230)\end{array}$ & $\begin{array}{l}0,661 * * * \\
(0,237)\end{array}$ & \\
\hline Edad & & $\begin{array}{l}0,019 * * * \\
(0,007)\end{array}$ & & & & & \\
\hline Nivel de ingresos & $x$ & $x$ & $x$ & $x$ & $x$ & & \\
\hline Nivel de estudios & $\begin{array}{l}-0,317^{* *} \\
(0,150)\end{array}$ & & & & & & \\
\hline Tamaño del municipio & & & $\begin{array}{l}-0,257^{* * *} \\
(0,093)\end{array}$ & & & $\begin{array}{l}-0,410 * * * \\
(0,153)\end{array}$ & \\
\hline Valoración de Fraga & $\begin{array}{l}0,505 * * * \\
(0,088)\end{array}$ & $x$ & $x$ & $\begin{array}{l}0,309 * * \\
(0,135)\end{array}$ & $x$ & $x$ & $x$ \\
\hline Valoración de Feijóo & $x$ & $x$ & $x$ & $x$ & $\begin{array}{l}0,652 * * * \\
(0,117)\end{array}$ & $\begin{array}{l}0,777 * * * \\
(0,110)\end{array}$ & $\begin{array}{l}0,662 * * * \\
(0,140)\end{array}$ \\
\hline Valoración de Beiras & $\begin{array}{l}-0,321 * * * \\
(0,084)\end{array}$ & $x$ & $x$ & $x$ & $x$ & $x$ & $x$ \\
\hline Valoración de Presedo & $\begin{array}{l}-0,292^{* * *} \\
(0,089)\end{array}$ & $x$ & $x$ & $x$ & $x$ & $x$ & $x$ \\
\hline Valoración Quintana & $x$ & $x$ & $x$ & $\begin{array}{l}-0,499 * * * \\
(0,131)\end{array}$ & $\begin{array}{l}-0,218 * * \\
(0,084)\end{array}$ & $x$ & $x$ \\
\hline Valoración Jorquera & $x$ & $x$ & $x$ & $x$ & $x$ & $\begin{array}{l}-0,587 * * * \\
(0,098)\end{array}$ & $x$ \\
\hline
\end{tabular}




\begin{tabular}{|c|c|c|c|c|c|c|c|}
\hline Variables & 1993 & 1997 & 2001 & 2005 & 2009 & 2012 & 2016 \\
\hline Valoración Leiceaga & $x$ & $x$ & $x$ & $x$ & $x$ & $x$ & $\begin{array}{l}-0,368 * * * \\
(0,121)\end{array}$ \\
\hline Valoración Losada & $x$ & $x$ & $x$ & $x$ & $x$ & $x$ & $\begin{array}{l}-0,245 * * \\
(0,118)\end{array}$ \\
\hline Valoración Rajoy & $x$ & $x$ & $x$ & $\begin{array}{l}0,651 * * * \\
(0,164)\end{array}$ & $\begin{array}{l}0,358 * * * \\
(0,097)\end{array}$ & $x$ & $x$ \\
\hline Valoración Zapatero & $x$ & $x$ & $x$ & $\begin{array}{l}-0,506 * * * \\
(0,129)\end{array}$ & $\begin{array}{l}-0,591 * * * \\
(0,096)\end{array}$ & $x$ & $x$ \\
\hline Simpatía PP & & $\mathrm{x}$ & $x$ & $\begin{array}{l}1,879 * * * \\
(0,382)\end{array}$ & $\begin{array}{l}1,439 * * * \\
(0,217)\end{array}$ & $\begin{array}{l}1,523 * * * \\
(0,226)\end{array}$ & $\begin{array}{l}1,438 * * * \\
(0,301)\end{array}$ \\
\hline $\begin{array}{l}\text { Valoración de la } \\
\text { campaña } \\
\text { de Fraga }\end{array}$ & $\begin{array}{l}0,729 * * * \\
(0,246)\end{array}$ & $\begin{array}{l}0,942 * * * \\
(0,142)\end{array}$ & $\begin{array}{l}1,461 * * * \\
(0,160)\end{array}$ & $x$ & $x$ & $x$ & $x$ \\
\hline Constante & $-6,219$ & $-11,301$ & $-10,211$ & $-15,889$ & $-7,882$ & $-11,507$ & $-12,915$ \\
\hline $\begin{array}{l}\text { R cuadrado de Nage- } \\
\text { lkerke }\end{array}$ & 0,885 & 0,782 & 0,783 & 0,947 & 0,904 & 0,913 & 0,913 \\
\hline $\begin{array}{l}\text { Logaritmo de la vero- } \\
\text { similitud }\end{array}$ & 200,197 & 489,824 & 591,967 & 133,915 & 316,499 & 264,525 & 128,031 \\
\hline
\end{tabular}

Fuente: elaboración propia a partir de datos del CIS.

\section{Conclusiones}

En la primera parte de la investigación nos hemos centrado en distintos aspectos de carácter descriptivo. Hemos abordado la ubicación espacial, tanto del PPdeG como de sus votantes, en el espacio definido por las escalas nacionalista e ideológica. A través, principalmente, de medias y de los gráficos de dispersión, hemos observado tres tendencias principales: a) los votantes del PP se han desplazado hacia posiciones más centrales dentro de la escala ideológica; b) los electores del PP se han ubicado cada vez más cerca del mínimo nacionalismo; y, 3) atendiendo a la ubicación subjetiva del PP en la escala nacionalista, los votantes populares, elección tras elección, han percibido al partido en posiciones más próximas al mínimo nacionalismo.

En la segunda parte del trabajo hemos entrado de lleno en la explicación de los componentes del voto al PP a través de dos modelos de regresión logística binaria. Los resultados que obtenemos los podemos sintetizar en los siguientes puntos: 
1) Observamos el papel fundamental que ha jugado la identificación partidista en la explicación del voto al PPdeG. Su presencia estable en los últimos encuentros electorales concuerda con el papel que la literatura le otorga a este componente, clásico por otro lado, en la explicación del comportamiento electoral. La simpatía por el PP ejerce, en todos los casos, un efecto positivo y de peso en las probabilidades de votar a la formación.

2) Constatamos la importancia de los cleavages. Son otro de los factores con mayor peso explicativo, especialmente la variable autoubicación en la escala ideológica, que persiste en todos y cada uno de los modelos. En este sentido, ubicarse en posiciones de derecha ha aumentado, en todas las elecciones autonómicas, las posibilidades de votar al PP. Sucede lo mismo con la variable autoubicación identitaria, aunque en este caso, su peso se ha difuminado con el paso de los años.

3) Relacionado con lo anterior, hemos observado dos hechos que aparecen en determinados momentos y que nos parecen paradójicos en el comportamiento de los electores del PP:

a. Mientras que la autoubicación en posiciones de derecha en la escala ideológica ejerce un efecto positivo en el voto a la formación popular, posicionar al PP a la derecha en esta misma escala, ejerce un efecto negativo sobre las probabilidades de votarlo.

b. Mientras que la autoubicación en posiciones de máximo nacionalismo en la escala nacionalista reduce las probabilidades de votar al PP, ubicar a la formación en posiciones de máximo nacionalismo, en la misma escala, las aumenta. Esto se puede relacionar con el propio carácter del galleguismo que ha creado el partido. Un discurso que se construye sobre el desprecio a la concepción nacionalista y que persigue la exaltación del sentimiento ya existente del ser gallego, basado en sus tradiciones y sus costumbres.

4) Con respecto a las variables contextuales, hemos observamos que la valoración a la Xunta de Galicia ha sido uno de los factores fundamentales en la explicación del voto a la formación popular, mientras que ni la valoración de la situación política ni la valoración de la situación económica — tanto en Galicia como en España- han resultado significativas en los modelos, lo que implica en parte la pérdida del peso del factor económico cuando este entra en conexión con otros componentes. En cualquier caso, la importancia de la valoración de la Xunta de Galicia se acrecienta al atender a la identificación que se produce entre el PP y la Administración autonómica después de décadas de gobierno. 
5) La valoración de los líderes, con especial fuerza los del PPdeG, ha explicado el hecho de votar a la formación en todas las elecciones ${ }^{17}$. Esto nos da cuenta del creciente peso que, de acuerdo con la literatura, juega la valoración de los líderes en la política contemporánea. En nuestro caso, la valoración positiva, tanto de Manuel Fraga como de Núñez Feijóo, ha ejercicio un efecto positivo en las probabilidades de votar al PP.

6) Las variables de naturaleza sociodemográfica han entrado de forma tímida, pero no despreciable, en la mayor parte de los modelos. Su presencia nos da la siguiente información: a) el aumento de la edad ha impactado de manera positiva sobre el voto al PP; b) el tamaño del municipio, medido en el número de habitantes, ha ejercido un efecto inverso en las probabilidades de votar a la formación; y, c) el nivel de ingresos y el nivel de estudios disminuyen las probabilidades de votar a los populares según aumentan.

\section{Bibliografía}

BARREIRO, Xosé Luís (2016). "Por qué Galicia llegó a ser un sitio distinto". En La era Feijóo. Elecciones gallegas 2016, coord. Manuel CHEDA y Luís LÓPEZ, 8-19. Santiago: A viva voz.

BERAMENDI, Justo. (2016). Historia mínima de Galicia. Madrid: Turner.

FRAGA, Manuel. (1987). En busca del tiempo servido. Barcelona: Editorial Planeta.

LAGARES, Nieves. (1999). Génesis y desarrollo del Partido Popular de Galicia. Madrid: Tecnos.

LAGARES, Nieves. (2003). "O Partido Popular de Galicia”. En Os partidos políticos en Galicia, coord. José Manuel RIVERA, 368-427. Vigo: Xerais.

LAGARES, Nieves. (2015). "Los componentes del voto". En Cataluña en proceso: las elecciones autonómicas de 2015, coord. José Manuel RIVERA, Juan MONTABES y Nieves LAGARES, 323-345. Valencia: Tirant Lo Blanch.

LAGARES, Nieves, María PEREIRA y José Manuel RIVERA (2018) "Diferencias y homogeneidades en el voto a Podemos y sus confluencias". En Las elecciones generales de 2015 y 2016, eds. Francisco LLERA, Juan MONTABLES y Montserrat BARAS. Madrid: CIS

LAGARES, Nieves, Erika JARÁIZ y María PEREIRA (2019) "Comportamiento electoral” En Gobierno y política en España. Juan MONTABES y Antonia MARTÍNEZ. Valencia: Tirant lo Blanch.

17. Hay que tener en cuenta que esta variable no estaba disponible en algunos modelos. 
LUGILDE, Anxo (2013) "O outono galego: as eleccións autonómicas (1981-2012)" En Análise das eleccións autonómicas galegas de 21 de outubro de 2012: reflexión sobre o sistema electoral, coord. Fernando MARTÍNEZ y Antonio TORRES DEL MORAL, 107-152. Santiago de Compostela: Andavira.

RIVERA, José Manuel, Nieves LAGARES, Alfredo CASTRO e Isabel DIZ (1999) "Las elecciones autonómicas en Galicia” En Os partidos políticos en Galicia, coord., Manuel ALCÁNTARA y Antonia MARTÍNEZ, 368-427. Vigo: Xerais.

RIVERA, José Manuel (2003) "Comportamento electoral e sistema de partidos en Galicia”. En Os partidos politicos en Galicia, coord., José Manuel Rivera, 368-427. Vigo: Xerais.

RIVERA, José Manuel y Erika JARÁIZ (2016) "Modelos de explicación del voto en las elecciones autonómicas catalanas de 2015" Revista Española de Ciencias Política, 42, 13-43. https://doi.org/10.21308/recp.42.01 